\title{
Immunopathogenesis of IBD: Batf as a Key Driver of Disease Activity
}

\author{
Kai Hildner ${ }^{a, b}$ Elise Punkenburg ${ }^{a, b}$ Benjamin Abendroth ${ }^{a, b}$ \\ Markus F. Neurath ${ }^{\mathrm{a}}$ \\ a Department of Medicine 1, University Hospital Erlangen, University of Erlangen-Nuremberg, Kussmaul Campus for \\ Medical Research, and bMax Eder Research Group Supported by the German Cancer Aid, Medical Department 1, \\ University Hospital Erlangen, Erlangen, Germany
}

\section{Key Words}

Batf · Th17 · Colitis · Inflammatory bowel diseases ·

Intestinal epithelial cell homeostasis

\begin{abstract}
Background: Inflammatory bowel diseases (IBDs) represent a group of chronic immune-mediated disorders that are influenced by a genetic predisposition and additional environmental triggers. Genome-wide association studies strongly implicate that a number of immune system-related genetic variations are critically contributing to the initiation and promotion of intestinal inflammation. Especially the identification of the strong association of a series of single nucleotide polymorphisms including interleukin (IL)-23R, CCR6, signal transducer and activator of transcription 3 (Stat3) and Stat4 with IBD susceptibility point at a critical involvement of $T$ cells and especially of IL-17a-producing Th17 cells in the immune pathogenesis of IBD. In line with this hypothesis, a series of preclinical studies have unequivocally established that $T$ cells are key drivers of immune-mediated colitis. Interestingly, especially Th17 cells were identified to be highly prevalent in inflamed IBD tissues, a finding that seems to be functionally relevant as genetic inactivation studies in the mouse resulted in almost complete suppression of colitis development. Key Messages: While targeting Th17 cell differentiation regulating transcription factors, as retinoic acidrelated orphan receptor gamma $\mathrm{t}(\mathrm{ROR} p \mathrm{t})$ is effective in pre-
\end{abstract}

venting murine colitis, one concern of drugs targeting ROR $\gamma \mathrm{t}$ in a clinical setting represents the large body of murine data unambiguously demonstrating that additional pathways within and outside the immune system are equally RORytdependent increasing the risk of undesirable side effects. The AP1 transcription factor Batf (B cell-activating transcription factor) appears to exclusively regulate pathways within lymphocytes. Importantly, Batf represents a central regulator of Th17 cell development and is strongly upregulated within IBD-affected tissues. Employing 2 acute colitis models, we demonstrate in this study that Batf-expressing $T$ cells are critical drivers of T cell-mediated colitis while in contrast to Stat3 loss of Batf does not affect intestinal epithelial cell homeostasis ex vivo. Conclusions: Targeting Batf in IBD emerges as an attractive therapeutic approach disabling colitogenic T cell activities while sparing off-target effects in the intestinal epithelial cell compartment.

(C) 2016 S. Karger AG, Basel

\section{Introduction}

Inflammatory bowel diseases (IBDs) mainly consist of 2 subtypes, Crohn's disease (CD) and ulcerative colitis (UC), and represent a chronic and frequently relapsing bowel disorder with an increasing incidence and prevalence in the western world [1]. IBD predominately affects the intestinal compartment while extraintestinal manifes-

\section{KARGER}

E-Mail karger@karger.com www.karger.com/ddi (c) 2016 S. Karger AG, Basel

0257-2753/16/0345-0040\$39.50/0
Prof. Dr. med. Kai Hildne

Department of Medicine 1

University Hospital Erlangen

Ulmenweg 18, DE-91054 Erlangen (Germany)

E-Mail Kai.Hildner@uk-erlangen.de 
tations as arthritis or cutaneous affections are also regularly observed. In conjunction with a complex genetic predisposition and largely unknown environmental factors (e.g. infections or food-born antigens), IBD is assumed to be an immune-mediated disorder [1-3]. However, while the initial pathogenetic steps underlying early-phase clinical disease manifestation are still poorly understood, disease-promoting effector mechanisms of established colitis has been thoroughly studied over the last decades leading to the identification of molecular targets that are now successfully employed in the therapeutic management of IBD during daily clinical routine [4]. Especially pro-inflammatory cytokines (e.g. tumor necrosis factor alpha) have been identified to be the key drivers and enhancers of intestinal inflammation consecutively resulting in tissue destruction, fibrosis and intestinal malfunction [4]. Accordingly, TNFa-blocking antibodies are widely distributed in the treatment regimen of IBD leading to excellent clinical responses in a substantial subgroup of IBD patients [5]. However, lack of mid-to-long-term control of inflammation within the majority of patients treated with $\mathrm{TNFa}$ blocking antibodies indicates astonishing and, until recently, less appreciated heterogeneity of the immune pathogenesis of IBD $[4,5]$. Since the immunological mechanisms and drivers underlying these different IBD 'phenotypes' are unknown and patients are not responsive to state-of-the-art therapies including TNFa blockade, the identification of pathways driving colitis in these subgroups and the development of innovative therapeutic intervention strategies beyond TNFa blockade are much needed in the future [5].

Besides innate immune cell-derived cytokine production by antigen-presenting cells, $\mathrm{T}$ cells are assumed to be the major producer of both pro-inflammatory as well as colitis-mitigating cytokines. T cells are widely accepted to represent a central effector cell population not only driving and promoting but also containing tissue manifestation in IBD [6]. Given a number of controversial study results, the role of T cells within IBD pathogenesis is complex and overall remains only partly understood [6]. However, T cells are frequently detected within colitic tissue in IBD patients and were shown to mediate colitis in a number of pre-clinical murine colitis models. Starting with the seminal studies by Mosmann and Coffman [7], T helper cells (Th cells) are divided into subgroups by the virtue of their cytokine expression profile (e.g., IFN-gamma producing Th1 cells and interleukin (IL)-4/IL-13 producing Th2 cells). Initially based on expression data and later on functional studies, CD was assumed to be largely driven by IFN-gamma producing Th1 cells, while UC was

Immunopathogenesis of IBD: Batf as a

Key Driver of Disease Activity largely characterized by IL- 5 and IL- 13 secreting Th 2 cells [4]. However, over the years, additional $\mathrm{T}$ cell subpopulations with more or less defined and restricted cytokine expression profiles have been identified and characterized also in the context of colitis. Among those, IL-17a-producing Th17, IL-9-producing Th9 and IL-22-producing Th22 cells have been implicated to be part of the increasingly complex, T cell-driven IBD pathogenesis [8-11]. While these novel $\mathrm{T}$ helper cell subpopulations are less stringently related to a certain IBD subtype, Th17 cells have been implied to be part of the immune pathogenesis underlying CD while Th22- and Th2-related Th9 cells are putatively contributing to the UC pathogenesis [9-11].

Substantial progress with respect to the understanding of the contribution of individual $\mathrm{T}$ helper cell subsets in the colitis pathogenesis has been made by the use of genetically modified mouse strains displaying hampered $\mathrm{T}$ cell functionality due to abrogated expression of transcriptional regulators that normally control $\mathrm{CD} 4^{+} \mathrm{T}$ helper cell differentiation. Interestingly, T-box transcription factor 21 (T-bet)-deficient $\mathrm{T}$ cells displaying hampered Th1 differentiation failed to promote colitis in vivo in support of the hypothesis that IFN-gamma producing Th1 cells are exerting colitis-promoting effects [12]. However, T cells either deficient of retinoic acid-related orphan receptor gamma $\mathrm{t}(\mathrm{ROR} \gamma \mathrm{t})$ or interferon regulatory factor 4 (IRF-4), both previously shown to control Th17 cell differentiation, similarly lacked colitis-inducing abilities suggesting that bona fide Th17 cells are context-dependent equally contributing to colitis manifestation [13-15]. However, inactivation or neutralization of individual Th17 cell-related cytokines like IL-17a were not effective and even exaggerated disease in some CD patients suggesting that alternative Th17 cell-related pathomechanisms drive colitis formation while IL-17a itself might even exert gut-protective effects $[14,16]$. In Th2-driven colitis models putatively modeling the pathogenesis of UC, transcription factor PU1-deficient T cells lacking Th9 differentiation abilities failed to promote Th2/Th9-dependent intestinal inflammation in vivo indicating that indeed Th9 cells are functionally relevant and a potential target for innovative treatment regimens in UC patients $[9,10]$. Collectively, results derived from murine studies firmly established that $\mathrm{T}$ helper cell subsets are critical promoters of colitis while molecular targeting of $\mathrm{T}$ cell differentiation regulating transcriptional factors might represent a potentially promising therapeutic approach in the future. Consistent with this assumption, a first proof of concept study was recently published demonstrating that allergen-induced allergic responses 
in patients were successfully modulated in vivo by targeting Th2 differentiation controlling transcription factor GATA3 via a GATA3-specific DNAzyme approach [17]. A phase IIa study (SECURE trial) evaluating the efficacy of a related agent in UC patients is under way. Given established roles of many transcription factors outside of the T lymphocyte compartment, off-target effects within other lineages and cell types, however, might limit the successful therapeutic application. Therefore, in addition to the strategy to administer the drug locally, molecules exerting its functions in a lymphocyte-restricted fashion might be most suitable to be selected as therapeutic targets to limit $\mathrm{T}$ cell-driven intestinal inflammation [5].

Previously, Batf, a member of the AP-1 family of transcription factors, was identified to be predominately expressed within the lymphocyte compartment $[18,19]$. Gene inactivation studies demonstrated that Batf controls Th17 cell differentiation both in vitro and in vivo as Batf-deficient mice lacked spontaneously occurring intestinal Th17 cells while Batf-deficient mice failed to $\mathrm{T}$ cell intrinsically mount $\mathrm{T}$ cell-driven neuro-inflammation in a mouse model of multiple sclerosis [18]. In striking contrast to the plethora of related Thelper cell differentiation regulating transcription factors [20-22], Batf seems to lack critical functions outside of $\mathrm{T}$ and $\mathrm{B}$ cells $[18,19]$. Interestingly, we recently reported that Batf is strongly upregulated in IBD-affected tissues in an inflammation-dependent fashion suggesting that Batf-expressing T cells are involved in the colitis pathogenesis [23]. To further address this, we studied the role of Batf in the context of intestinal inflammation in mice in more detail. Employing an ex vivo approach and 2 independent acute colitis models, we provide evidence that Batf-dependent $\mathrm{T}$ cells are exerting pro-inflammatory functions in a $\mathrm{T}$ cell-intrinsic manner while Batf expression within the intestinal epithelial cell compartment seems to be functionally redundant. Collectively, our data indicate that targeted inhibition of Batf might represent a promising, $\mathrm{T}$ cell-directed approach to control IBD in the future.

\section{Results}

Given the established key role of Batf in Th17 cell differentiation, upregulated Batf expression within colitic tissue of both CD and UC patients in an inflammationdependent manner as observed previously is suggestive to result in an increased influx of lymphocytes into the bowel wall [23]. However, related transcription factors like signal transducer and activator of transcription 3 (Stat3) or hypoxia-inducible factor 1-alpha cooperating with Batf during Th17 cell differentiation and hence seemingly context-dependent functionally related were shown to exert additional roles including intestinal epithelial cells overall complicating the interpretation of gene expression data derived from whole gut tissue specimen $[20,24]$. To further explore whether Batf is involved in intestinal epithelial cell homeostasis in an epithelial cell-intrinsic manner, we performed intestinal crypt culture experiments adopting a previously published ex vivo culture system [25]. Here, intestinal crypts are cultured in the presence of a defined cocktail of growth and differentiation inducing factors resulting in the formation of socalled organoids that originate from intestinal stem cells residing at the bottom of the crypts and display all cell types regularly present in the intestinal epithelial cell compartment including goblet and Paneth cells [25]. Accordingly, Batf ${ }^{+/+}$and $\mathrm{Batf}^{-/-}$intestinal crypts were isolated, and organoid cultures were initiated and followed over the next days as organoids started to form. Importantly, kinetics and dynamics of organoid development occurred in a Batf expression-independent manner (fig. 1a). In addition, morphologic features as well as the rate of apoptotic cell death observed in these cultures over time were indistinguishable between Batf-sufficient and Batf-deficient organoid cultures (fig. 1a, and data not shown). In addition, spontaneous expression levels of anti-microbial molecules like angiogenin (Ang4) and defensing beta 3 (DefB3) representing signature molecules of specialized intestinal epithelial cells (e.g., Paneth cells) were also independent of Batf expression (fig. 1b). Finally, IL-17a expression within organoids was uncompromised in the absence of Batf overall indicating that development, morphology and functionality of intestinal organoids is not regulated by Batf in the steady state (fig. 1c).

The integrity and functionality of intestinal epithelial cells in the context of massive immune cell activation as observed in IBD patients have been increasingly recognized to be a crucial component of the intestinal homeostasis while dysregulated or even abrogated epithelial cell homeostasis is implicated in the initiation and/or promotion of immune-mediated IBD [26]. Therefore, we sought to assess whether mimicking immune cell activation in response to a pathogen would have an impact on morphology and gene expression profiles in a Batf-dependent manner. To test this, we added a series of inflammatory stimuli to the cultures for $30 \mathrm{~h}$. Interestingly, TNFa induced the highest rate of apoptosis when compared to IL-6 and poly-IC that represents a synthetic immune stimulant mimicking double-stranded viral RNA. How- 


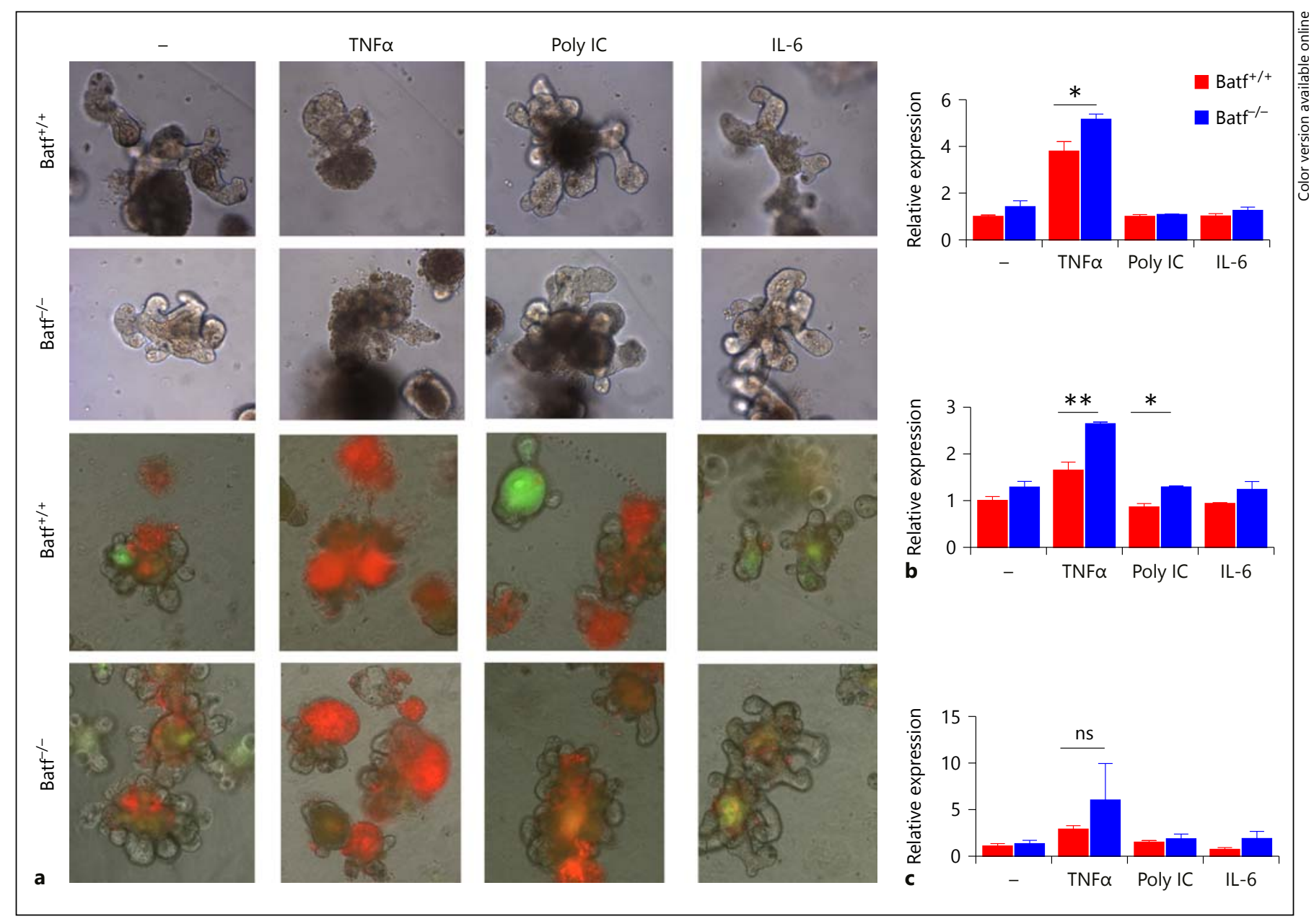

Fig. 1. Loss of Batf expression within intestinal epithelial progenitor cells is dispensable for epithelial cell homeostasis ex vivo (a) Light microscopy (upper 2 rows) and fluorescence microscopy (lower 2 rows) images of small intestinal organoids from $\mathrm{Batf}^{+/+}$and $\mathrm{Batf}^{-/-}$ mice. For the final $30 \mathrm{~h}$ of culture, organoids remained untreated (-) or were stimulated with TNFa, poly IC or IL-6 as indicated. Red: propidium iodide (dead cells). Green: calcein-AM (viable cells). b, c Small intestinal organoids derived from Batf ${ }^{+/+}$and Batf ${ }^{-1-}$ mice remained untreated (-) or were stimulated with indicated stimuli (TNFa, poly IC or IL-6) for the last $30 \mathrm{~h}$ of culture. After harvesting whole tissues, RNA was isolated, cDNA was generated and qPCR analyses for Ang4 (b, top panel), Defb3 (b, bottom panel) and IL$17 \mathrm{a}$ (c) were performed. Displayed gene expression levels within organoid tissue of indicated genotypes represent normalized, relative fold of expression compared to the expression that was detected in untreated (-) Batf ${ }^{+/+}$samples arbitrarily set to an expression level of 1 . Bar graphs represent mean values \pm SEM derived from 3 independent samples of one representative experiment. For statistical analyses, an unpaired, 2-sided Student's t test was performed. $\mathrm{ns}=$ Not significant; ${ }^{*} \mathrm{p}<0.05 ;{ }^{* *} \mathrm{p}<0.01$.

ly-IC-induced effects on Ang4, and IL-17a expression was Batf independent while expression of Defb3 showed a slim yet significant increase in the absence of Batf. In summary, development, morphology and steady state functionality of ex vivo intestinal organoids are Batf independent while TNFa and to a lesser extent poly-IC- but not IL-6-induced expression of anti-microbial molecules are increased in the absence of Batf.

To functionally assess the contribution of Batf during colitis in vivo, we employed 2 commonly used colitis 
models: the dextran sodium sulphate (DSS) and the T cell transfer colitis model $[27,28]$. While the earlier is based on a DSS-mediated epithelial layer injury with consecutive diapedesis of commensals initiating a pro-inflammatory immune response and is assumed to induce colitis in the absence of $\mathrm{T}$ cells, the $\mathrm{T}$ cell transfer colitis model is based on the transplantation of naive $\mathrm{CD} 4^{+} \mathrm{CD} 25^{-}$congenic $\mathrm{T}$ cells into lymphopenic Rag1 $1^{-/-}$or Rag $2^{-/-}$mice resulting in $\mathrm{T}$ cell expansion, differentiation and ultimately development of a systemic inflammatory immune response also affecting the intestinal compartment [27, 28]. First, Batf ${ }^{+/+}$and Batf $^{-/-}$mice were treated with $1.5 \%$ DSS in the drinking water over 7 days followed by regular water administration. As shown in figure 2a, both groups displayed an indistinguishable yet mild body weight reduction over time compared to day 0 . At day 14, that is 7 days after DSS discontinuation, colitis severity was assessed by mini-endoscopic analysis, and intestinal inflammation in both $\mathrm{Batf}^{+/+}$and $\mathrm{Batf}^{-/-}$mice was scored employing a modified murine endoscopic index of colitis severity (MEICS) $[23,29]$. Here, Batf ${ }^{+/+}$mice showed a mild-to-moderate colitis activity as indicated by a mean MEICS score of about 5 that was overall indistinguishable from colitis severity observed in $\mathrm{Batf}^{-/-}$mice (fig. 2b). Collectively, Batf deficiency does not lead to an increase or decrease of acute signs of intestinal inflammation in the DSS-induced colitis model.

We then employed the $\mathrm{T}$ cell transfer colitis model which represents the most established colitis model due to its $\mathrm{T}$ cell dependence and involvement of the small intestine as observed in CD patients [30]. In line with results obtained in an independent study [31], Batf-deficient and, hence, intrinsically Th17-deficient $\mathrm{T}$ cells failed to induce systemic and intestinal inflammation while $\mathrm{Batf}^{+/+}$ $T$ cells expectedly led to an increasing loss of body weight starting around 20 days past $\mathrm{T}$ cell transfer, and mice receiving Batf ${ }^{-/-} \mathrm{T}$ cells did not show any body weight alterations over time (fig. 2c). Additionally, endoscopic evaluation of the distal colon revealed that $\mathrm{Batf}^{+/+}$but not Batf $^{-1-}$ T cells induced severe colitis (fig. $2 \mathrm{~d}$ ).

Given Batf expression-independent acute DSS-induced colitis manifestation (fig. $2 \mathrm{a}$ and $\mathrm{b}$ ) and our previous observation that DSS application in the context of colon tumor formation induced by azoxymethane largely reconstituted endogenous $\mathrm{T}$ helper cell numbers in the lamina propria of $\mathrm{Batf}^{-/-}$mice [23], we hypothesized that concomitant and transient DSS-induced mucosal inflammation might rescues the hampered colitogenic potential of Batf $^{-1-} \mathrm{T}$ cells. Therefore, we transferred naïve $\mathrm{CD} 4{ }^{+} \mathrm{CD} 25^{-} \mathrm{T}$ cells derived from Batf $\mathrm{B}^{+/+}$or $\mathrm{Batf}^{-/-}$donors into Rag $1^{-/-}$mice at day 0 and treated both groups of mice with $1.5 \%$ DSS in the drinking water over 7 days. Within the first 2 weeks after initiation, we did not detect any T cell source-dependent differences in the body weight development which is consistent with an unaltered acute DSS-induced colitis development as described above. However, in the third week after T cell transfer, Batf ${ }^{+/+} \mathrm{T}$ cell receiving $\operatorname{Rag} 1^{-/-}$mice started to show a slightly reduced body weight compared to $\mathrm{Batf}^{-/-} \mathrm{T}$ cell receiving mice indicating the initiation of $\mathrm{T}$ cell-mediated colitis by Batf-expressing T cells in this model (fig. 2e). More importantly, endoscopic evaluation of the distal colon confirmed significantly increased colitis activity in Batf ${ }^{+/+} \mathrm{T}$ cell receiving mice while $\mathrm{Batf}^{-/-} \mathrm{T}$ cells remained unable to induce equivalently enhanced colitis activity despite previous induction of DSS-induced colitis (fig. 2f).

\section{Discussion and Conclusions}

In this study, we investigated the role of the AP1 transcription factor Batf in 2 acute colitis models. The results shown here clearly demonstrate that Batf-expressing T cells are key drivers of T cell-mediated colitis in mice. However, in the DSS-induced colitis model characterized by a largely lymphocyte-independent immune pathogenesis, Batf deficiency does not protect against clinical manifestation of acute colitis. Simultaneously initiated DSS-induced colitis did not rescue hampered $\mathrm{T}$ cell-driven colitis development potentially due to a failure to overcome Batf-dependent upregulation of gut homing receptors by transferred $\mathrm{T}$ cells as previously reported [31]. In addition, ex vivo studies demonstrated that intestinal epithelial stem cells are able to develop into organoids displaying largely unaltered intestinal epithelial cell development and differentiation despite the absence of Batf. Overall, this study collectively suggests that Batf represents a promising molecular target that may allow selective inhibition of $\mathrm{T}$ cell-driven intestinal tissue inflammation while detrimental effects within the intestinal epithelial cell compartment are not induced.

Consistent with our results and the known role for Batf in Th17 differentiation, Th17 cells have been implicated to be a critical driver of colitis both in murine and human settings [ 18,23$]$. First, Th 17 cells are frequently detectable in colitic tissues implying a functional contribution in the immune pathogenesis [8]. Second, multiple studies using genetically modified T cells with hampered Th17 cell differentiation due to the deletion of transcriptional regulators clearly demonstrated the colitis-promoting abilities of Th17 cells [14, 32-34]. However, caution has to be ex- 


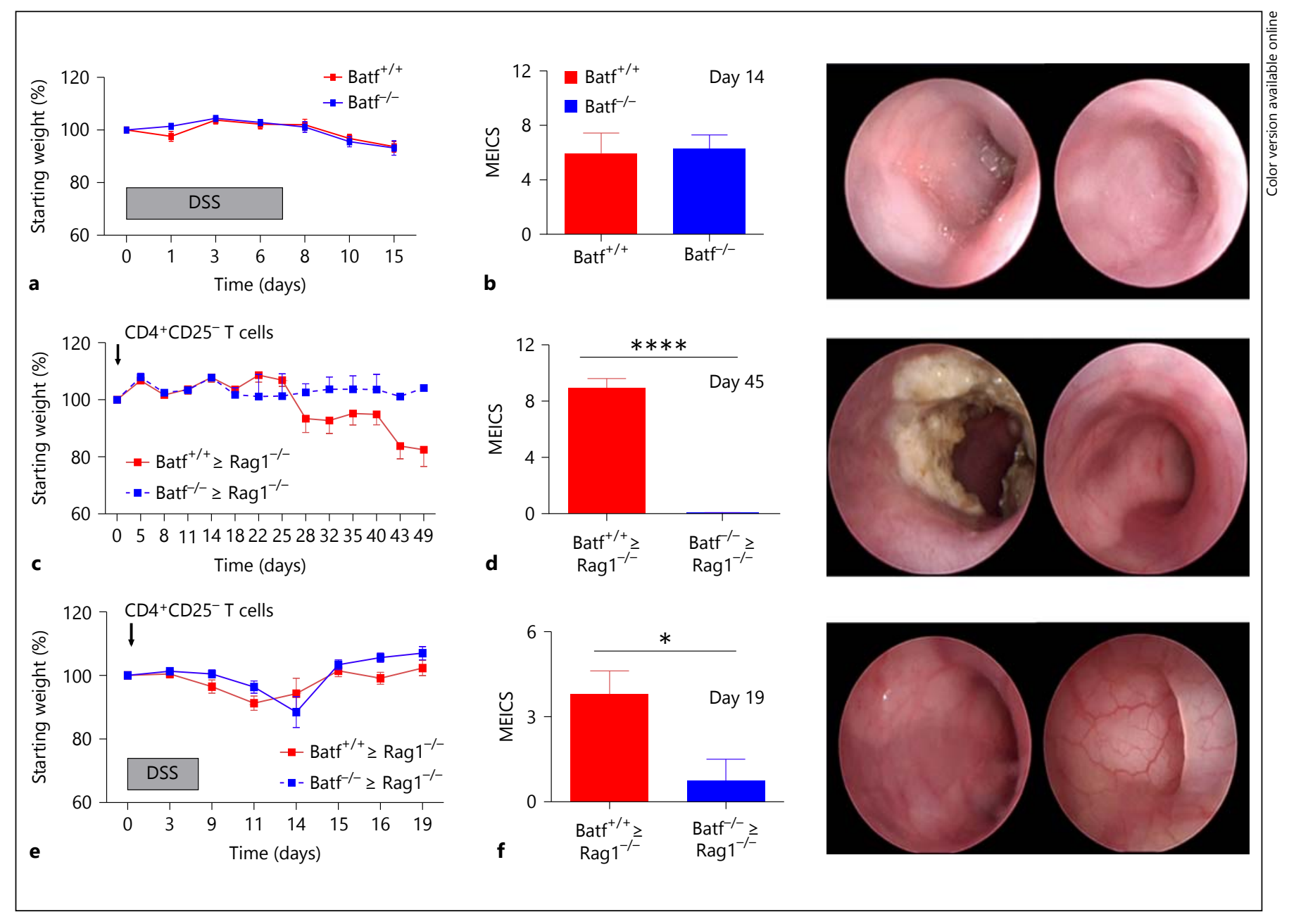

Fig. 2. Batf promote acute colitis in a $\mathrm{T}$ cell-intrinsic manner. a, b Eight to 12-week-old $\mathrm{Batf}^{+/+}$and $\mathrm{Batf}^{-/-} \mathrm{C} 57 / \mathrm{Bl} 6$ mice were treated with $1.5 \%$ DSS in the drinking water as indicated (filled box). After 7 days, DSS-containing drinking water was switched to regular water. Body weight of mice was repeatedly assessed and is displayed in (a) in percentage of the starting body weight at day 0 $(100 \%)$. At day 14 , as shown in (b), colitis activity was determined by mini-endoscopic evaluation of the distal colon (representative images at the right) and was quantified by assessing several macroscopic colitis criteria (thickening of the bowel wall, changes of the vascularity, granularity of the mucosal layer and consistency of the stool) as summarized in the shown modified MEICS scoring system (left panel). Bar graphs represent mean values \pm SEM derived from at least 5 independent mice of one representative experiment. c, d $10^{6}$ magnetically purified $\mathrm{CD} 4^{+} \mathrm{CD} 25^{-}$naïve $\mathrm{Batf} \mathrm{f}^{+/+}$ and Batf $^{-1-} \mathrm{T}$ cells with a purity of $>95 \%$ were transferred into Rag $1^{-1-}$ mice via i.p. injection at day 0 as indicated. Body weight of mice was repeatedly assessed and is displayed in (c) in percentage of the starting body weight at day $0(100 \%)$. At day 45 post $\mathrm{T}$ cell transfer, as shown in (d), colitis activity was determined by miniendoscopic evaluation of the distal colon (representative images at the right) and was quantified by assessing several macroscopic colitis criteria as described above summarized in the shown modified MEICS scoring system (left panel). Bar graphs represent mean values \pm SEM derived from at least 5 independent mice of one representative experiment. For statistical analyses, an unpaired, 2-sided Student's t test was performed. ${ }^{* * * *} \mathrm{p}<0.0001$. e, f $10^{6}$ magnetically purified $\mathrm{CD} 4^{+} \mathrm{CD} 25^{-}$naïve $\mathrm{Batf}^{+/+}$and $\mathrm{Batf}^{-/-}$ $\mathrm{T}$ cells with a purity of $>95 \%$ were transferred into $\mathrm{Rag}^{-/-}$mice via i.p. injection at day 0 as indicated. In addition, mice were kept on $1.5 \%$ DSS containing drinking water until day 7 followed by switching to regular drinking water. Body weight of mice was repeatedly assessed and is displayed in (e) in percentage of the starting body weight at day $0(100 \%)$. At day 19 post T cell transfer, as shown in (f), colitis activity was determined by mini-endoscopic evaluation of the distal colon (representative images at the right) and was quantified by assessing several macroscopic colitis criteria as described above and as summarized in the shown modified MEICS scoring system (left panel). Bar graphs represent mean values \pm SEM derived from at least 5 independent mice of one representative experiment. For statistical analyses, an unpaired, 2-sided Student's t test was performed. ${ }^{*} \mathrm{p}<0.05$. 
ercised when the results of these studies are interpreted as many transcriptional regulators are nowadays less referred to be master regulators of individual lineage decisions but appear to exert multiple regulatory roles within different lymphocyte differentiation processes [35]. Hence the same transcription factor can act in multiple lineages as a lineage-specifying factor and hence modulate several lineages as shown, for example, IRF-4 in Th2, Th9, Th17 and induced regulatory $\mathrm{T}$ cell differentiation [36-39]. Similarly, Batf was shown to be involved in the regulation of multiple $\mathrm{CD} 4^{+} \mathrm{T}$ helper lineages including Th9, Th17, follicular T helper cell and fat tissue resident regulatory $\mathrm{T}$ cell differentiation overall resembling results obtained with T cell studies in IRF-4-deficient mice [18, 40-42]. In fact, for many of those instances, the molecular cooperativity of Batf and IRF-4 during regulation of specific, lineage-related target genes (e.g., in Th17 cells) has been experimentally proven [42-45]. However, in contrast to IRF-4, a direct role of Batf in Th2 differentiation is controversially discussed although $\mathrm{Th} 2$ responses in vivo are hampered in the absence of Batf as observed in a murine asthma model $[31,39,45,46]$. In addition, Batf was shown to control anti-viral $\mathrm{CD}^{+} \mathrm{T}$ cell responses while Batf deficiency also controls class switch recombination in $\mathrm{B}$ lymphocytes again resembling effects observed in IRF-4 $4^{-/-}$mice $[40,47,48]$.

Collectively, the tissue-wide expression profiles of Batf and so far reported functional data imply that Batf-regulated pathways reside within the $\mathrm{T}$ and $\mathrm{B}$ lymphocyte compartments $[18,19,23,31,40,41,48,49]$. In contrast to Batf, the majority of related Th17 fate-defining factors, however, at least in the murine system also control pathways within non-lymphocytes as shown, for example, Tbet, IRF-4, Stat3, Hif1-alpha and ROR $y$ t in dendritic cell, natural killer cell, innate lymphoid cell and regulatory $\mathrm{T}$ cell development or functionality, respectively [21, 24, 50-52]. In addition, Stat 3 plays a pivotal role in intestinal epithelial cell homeostasis and functionality suggesting that Stat3 inhibition might broadly affect both lymphocytes, non-lymphocytic immune cell populations and also non-hematopoietic host cells like intestinal epithelial cells [20]. Hence, given our previously published results showing unaltered functionality of IL-17a producing non-lymphocytes in the absence of Batf [23] and our current report showing largely indistinguishable intestinal epithelial cell differentiation and functionality irrespective of the Batf expression status, therapeutic inhibition of Batf within the intestinal compartment appears in delineation of related Th17 fate-regulating transcription factors to functionally block predominately pathways within lymphocytes while undesired blocking of Batf within non-lymphocytes and intestinal epithelial cells might result only in few, if any, unwanted off-target effects.

Together, this study provides crucial evidence for a critical role of Batf during $\mathrm{T}$ cell-mediated colitis and might inspire to further investigate the therapeutic efficacy and safety of blocking Batf-driven pathways within the immune pathogenesis underlying IBD.

\section{Disclosure Statement}

The authors disclose no conflicts.

\section{Author Contributions}

E.P. and B.A. performed experiments, analyzed and interpreted data. M.F.N. designed experiments, gave critical advice and helped with the interpretation and critical discussion of the results. K.H. designed and directed the study and performed analyses. K.H. and M.F.N. wrote the manuscript.

\section{Acknowledgments}

We thank T. Vogler at the University Hospital Erlangen for excellent technical assistance. We thank Dr. Claudia Günther and Dr. Eva Martini (both Medical Department 1, University Hospital Erlangen) for substantial scientific support in setting up the organoid culture model system.

\section{Funding}

This study was supported by the Max-Eder Research Program of the German Cancer Aid (K.H.); the ELAN program (project $\#$ 09.08.19.1 to K.H.) of the University Erlangen-Nuremberg (K.H.); the Interdisciplinary Center for Clinical Research Erlangen (project A53 to K.H.); and the Collaborative Research Center 1181 (SFB 1181 of the DFG, projects B02 (M.F.N.) and B05 (K.H.), University Hospital Erlangen, Friedrich-Alexander University Erlangen-Nuremberg.

References

1 Strober W, Fuss I, Mannon P: The fundamental basis of inflammatory bowel disease. J Clin Invest 2007;117:514-521.

2 Duerr RH, et al: A genome-wide association study identifies IL23R as an inflammatory bowel disease gene. Science 2006;314:1461-1463.

3 Raine T, Liu JZ, Anderson CA, Parkes M, Kaser A: Generation of primary human intestinal $\mathrm{T}$ cell transcriptomes reveals differential expression at genetic risk loci for immune-mediated disease. Gut 2015;64:250-259.

4 Neurath MF: Cytokines in inflammatory bowel disease. Nat Rev Immunol 2014;14:329-342.
46

Dig Dis 2016;34(suppl 1):40-47 DOI: $10.1159 / 000447281$ 
5 Neurath MF: New targets for mucosal healing and therapy in inflammatory bowel diseases. Mucosal Immunol 2014;7:6-19.

6 Chen ML, Sundrud MS: Cytokine networks and T-cell subsets in inflammatory bowel diseases. Inflamm Bowel Dis 2016;22:11571167.

7 Mosmann TR, Coffman RL: Heterogeneity of cytokine secretion patterns and functions of helper T cells. Adv Immunol 1989;46:111147.

8 Jiang W, et al: Elevated levels of Th17 cells and Th17-related cytokines are associated with disease activity in patients with inflammatory bowel disease. Inflamm Res 2014;63:943-950.

9 Gerlach K, et al: TH9 cells that express the transcription factor PU.1 drive T cell-mediated colitis via IL-9 receptor signaling in intestinal epithelial cells. Nat Immunol 2014;15: 676-686.

10 Nalleweg N, et al: IL-9 and its receptor are predominantly involved in the pathogenesis of UC. Gut 2015;64:743-755.

11 Leung JM, et al: IL-22-producing CD4+ cells are depleted in actively inflamed colitis tissue. Mucosal Immunol 2014;7:124-133.

12 Neurath MF, et al: The transcription factor Tbet regulates mucosal $\mathrm{T}$ cell activation in experimental colitis and Crohn's disease. J Exp Med 2002;195:1129-1143.

13 Ivanov II, et al: The orphan nuclear receptor RORgammat directs the differentiation program of proinflammatory IL-17+ T helper cells. Cell 2006;126:1121-1133.

14 Leppkes $M$, et al: RORgamma-expressing Th17 cells induce murine chronic intestinal inflammation via redundant effects of IL-17A and IL-17F. Gastroenterology 2009;136:257267.

15 Mudter J, et al: The transcription factor IFN regulatory factor-4 controls experimental colitis in mice via T cell-derived IL-6. J Clin Invest 2008;118:2415-2426.

16 Hueber W, et al: Secukinumab, a human antiIL-17A monoclonal antibody, for moderate to severe Crohn's disease: unexpected results of a randomised, double-blind placebo-controlled trial. Gut 2012;61:1693-1700.

17 Krug N, et al: Allergen-induced asthmatic responses modified by a GATA3-specific DNAzyme. N Engl J Med 2015;372:1987-1995.

18 Schraml BU, et al: The AP-1 transcription factor Batf controls $\mathrm{T}(\mathrm{H}) 17$ differentiation. Nature 2009;460:405-409.

19 Tussiwand R, et al: Compensatory dendritic cell development mediated by BATF-IRF interactions. Nature 2012;490:502-507.

20 Bollrath J, et al: gp130-mediated Stat 3 activation in enterocytes regulates cell survival and cell-cycle progression during colitis-associated tumorigenesis. Cancer Cell 2009;15:91102.
21 Schlitzer A, et al: IRF4 transcription factordependent $\mathrm{CD} 11 \mathrm{~b}+$ dendritic cells in human and mouse control mucosal IL-17 cytokine responses. Immunity 2013;38:970-983.

22 Eberl G, Littman DR: Thymic origin of intestinal alphabeta $T$ cells revealed by fate mapping of RORgammat+ cells. Science 2004;305: 248-251.

23 Punkenburg E, et al: Batf-dependent Th17 cells critically regulate IL-23 driven colitis-associated colon cancer. Gut 2016;65:11391150.

24 Kelly CJ, et al: Fundamental role for HIF-1a in constitutive expression of human $\beta$ defensin-1. Mucosal Immunol 2013;6:11101118

25 Sato T, et al: Single Lgr 5 stem cells build cryptvillus structures in vitro without a mesenchymal niche. Nature 2009;459:262-265.

26 Peterson LW, Artis D: Intestinal epithelial cells: regulators of barrier function and immune homeostasis. Nat Rev Immunol 2014; 14:141-153.

27 Wirtz S, Neufert C, Weigmann B, Neurath MF: Chemically induced mouse models of intestinal inflammation. Nat Protoc 2007;2: 541-546.

28 Mottet C, Uhlig HH, Powrie F: Cutting edge: cure of colitis by CD4+CD25+ regulatory $\mathrm{T}$ cells. J Immunol 2003;170:3939-3943.

29 Becker C, Fantini MC, Neurath MF: High resolution colonoscopy in live mice. Nat Protoc 2006;1:2900-2904.

30 Wirtz S, Neurath MF: Mouse models of inflammatory bowel disease. Adv Drug Deliv Rev 2007;59:1073-1083.

31 Wang C, et al: BATF is required for normal expression of gut-homing receptors by $\mathrm{T}$ helper cells in response to retinoic acid. J Exp Med 2013;210:475-489.

32 Ciofani $M$, et al: A validated regulatory network for Th17 cell specification. Cell 2012; 151:289-303.

33 Mudter J, et al: IRF4 regulates IL-17A promoter activity and controls ROR $\gamma \mathrm{t}$-dependent Th17 colitis in vivo. Inflamm Bowel Dis 2011; 17:1343-1358.

34 Yang XO, et al: STAT3 regulates cytokinemediated generation of inflammatory helper T cells. J Biol Chem 2007;282:9358-9363.

35 Oestreich KJ, Weinmann AS: Master regulators or lineage-specifying? Changing views on CD4+ T cell transcription factors. Nat Rev Immunol 2012;12:799-804.

36 Bollig N, et al: Transcription factor IRF4 determines germinal center formation through follicular T-helper cell differentiation. Proc Natl Acad Sci U S A 2012;109:8664-8669.

37 Brüstle A, et al: The development of inflammatory $\mathrm{T}(\mathrm{H})-17$ cells requires interferon-regulatory factor 4. Nat Immunol 2007;8:958966.
38 Staudt V, et al: Interferon-regulatory factor 4 is essential for the developmental program of T helper 9 cells. Immunity 2010;33:192-202.

39 Rengarajan J, et al: Interferon regulatory factor 4 (IRF4) interacts with NFATc2 to modulate interleukin 4 gene expression. J Exp Med 2002;195:1003-1012.

40 Ise $\mathrm{W}$, et al: The transcription factor BATF controls the global regulators of class-switch recombination in both B cells and T cells. Nat Immunol 2011;12:536-543.

41 Jabeen R, et al: Th9 cell development requires a BATF-regulated transcriptional network. J Clin Invest 2013;123:4641-4653.

42 Vasanthakumar A, et al: The transcriptional regulators IRF4, BATF and IL-33 orchestrate development and maintenance of adipose tissue-resident regulatory T cells. Nat Immunol 2015;16:276-285

43 Glasmacher E, et al: A genomic regulatory element that directs assembly and function of immune-specific AP-1-IRF complexes. Science 2012;338:975-980.

$44 \mathrm{Li}$ P, et al: BATF-JUN is critical for IRF4-mediated transcription in T cells. Nature 2012; 490:543-546.

45 Tussiwand R, et al: Compensatory dendritic cell development mediated by BATF-IRF interactions. Nature 2012;490:502-507.

46 Übel C, et al: The activating protein 1 transcription factor basic leucine zipper transcription factor, ATF-like (BATF), regulates lymphocyte- and mast cell-driven immune responses in the setting of allergic asthma. J Allergy Clin Immunol 2014;133:198-206.e1e9.

47 Betz BC, et al: Batf coordinates multiple aspects of $B$ and $T$ cell function required for normal antibody responses. J Exp Med 2010; 207:933-942.

48 Kurachi $\mathrm{M}$, et al: The transcription factor BATF operates as an essential differentiation checkpoint in early effector CD8+ T cells. Nat Immunol 2014;15:373-383.

49 Murphy TL, Tussiwand R, Murphy KM Specificity through cooperation: BATF-IRF interactions control immune-regulatory networks. Nat Rev Immunol 2013;13:499-509.

50 Sawa S, et al: ROR $\gamma \mathrm{t}+$ innate lymphoid cells regulate intestinal homeostasis by integrating negative signals from the symbiotic microbiota. Nat Immunol 2011;12:320-326.

51 Gordon SM, et al: The transcription factors T-bet and Eomes control key checkpoints of natural killer cell maturation. Immunity 2012;36:55-67.

52 Lugo-Villarino G, Maldonado-Lopez R, Possemato R, Penaranda C, Glimcher LH: T-bet is required for optimal production of IFNgamma and antigen-specific $\mathrm{T}$ cell activation by dendritic cells. Proc Natl Acad Sci U S A 2003;100:7749-7754
Immunopathogenesis of IBD: Batf as a

Key Driver of Disease Activity
Dig Dis 2016;34(suppl 1):40-47

DOI: $10.1159 / 000447281$ 